\title{
EL CULTO JOVEN EN LA ASOCIACIÓN PERUANA CENTRAL ESTE: LIMA 2015
}

\author{
Benjamín Rojas Yauri, Jesús Hanco Torres, \\ Cristian Gonzales Yupanqui, Felipe Esteban Silva \\ benjamin@upeu.edu.pe \\ Universidad Peruana Unión
}

El objetivo general de esta investigación es identificar la condición de los programas del culto joven en la APCE, Lima 2015. Los objetivos especificos son describir la condición de la alabanza, oración, testimonio y mensaje en los programas del culto joven en la APCE, Lima 2015. Para alcanzar este conocimiento se utilizó un sistema de encuestas que responda al objetivo general y a los objetivos especificos de esta investigación que por su tipo es descriptiva exploratoria que, además, tiene un enfoque cuantitativo y que, por su diseño, es transversal no experimental. Las encuestas fueron dirigidas a 70 iglesias de la APCE y se aplicaron un total de 900 encuestas de las cuales fueron consideradas 428 , el resto fue desechado por presentar desperfectos para su interpretación y lectura. Finalmente, los resultados demuestran la importancia que tiene para los participantes del Culto Joven en la APCE, la inclusión de la alabanza, oración, testimonio y mensaje planificados e integrados por un eje o modelo.

Palabras clave: Culto joven, alabanza, oración, testimonio, mensaje, APCE. 


\title{
YOUNG WORSHIP IN THE CENTRAL EAST PERUVIAN CONFERENCE: LIMA 2015
}

\begin{abstract}
The general objective of this research is to identify the condition of the young cult programs at "APC-Este" in Lima, 2015. The specific objectives are to describe the condition of praise, prayer, testimony and missionary programs in young's cults programs. To achieve this knowledge a survey system that meets the general objective and specific objectives of this research was used. It is an exploratory descriptive type, which also has a quantitative approach, and by design is transversal non-experimental. The surveys were conducted at 70 "APC-Este" churches, this surveys were applied a total of 900 of which 428 were considered, the rest was scrapped by present damages in their interpretation and reading. Finally, the results demonstrate the importance for participants in the "ACP-Este" Young Cults including worship, prayer, testimony and message that is planned and integrated by a shaft or model.
\end{abstract}

Keywords: Young Worship, Praise, Prayer, Mission, Message, APC-Este. 


\section{Introducción}

La Iglesia Adventista del Séptimo Día (IASD) tuvo sus inicios con jóvenes visionarios e intrépidos, pero sobre todo consagrados a la causa de Dios. Ellen White, John Loughborough, J. N. Andrews, Uriah Smith y John Harvey Kellogg hicieron un significativo impacto desde sus años más jóvenes.

En la actualidad los jóvenes continúan siendo el eje que mueve a la Iglesia, pues más del $50 \%$ de los feligreses de la IASD se encuentra entre los 16 y 40 años de edad. ${ }^{1}$ Esta realidad hizo que hace más de 125 años atrás, fuese creado por jóvenes y para jóvenes el Ministerio Joven de la IASD, hoy son más de 10 millones, los que hacen parte de esta red y, por la gracia y obra de Dios, este número irá en aumento.

El último informe, dado por la IASD en Sudamérica, confirma la realidad que se viene presen-

\footnotetext{
${ }^{1}$ Adventist.org "Remember your creator" http://www.adventist.org/en/ vitality/youth/
}

tando, pues el $62 \%$ de los miembros tiene menos de 40 años y solo el 38\% de la feligresía tiene 41 años o más. ${ }^{2}$ Es decir, la gran mayoría de la Iglesia son jóvenes y son estos los que enfrentan desafíos mayores cada día, pues la sociedad y cultura que los rodea puede sacarlos de su posición inicial y colocarlos en una posición extraña a su razón de ser. Por eso la Iglesia unida como una familia, donde miembros jóvenes y adultos se interrelacionan, trabaja de forma práctica para desarrollar en los jóvenes adventista del presen-

${ }^{2} \mathrm{El}$ cuadro estadístico presentado por la secretaría de la División Sudamericana de la Iglesia Adventista del Séptimo Día el 3 de noviembre del 2015, en ocasión del Concilio Quinquenal es el siguiente:

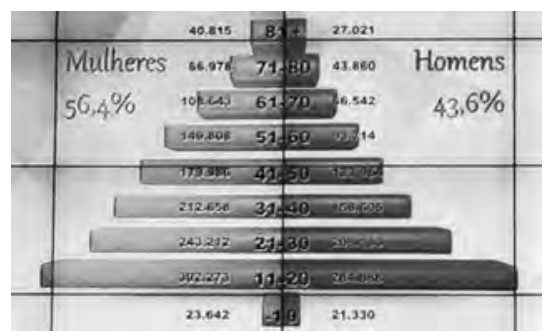

Para mayor información, se puede visitar la siguiente dirección electrónica http://noticias.adventistas.org/es/noticia/institucional/en-1915-la-iglesia-adventista-tenia-solo-88-templos-en-sudamerica/ 
te el mismo espíritu de aquellos que iniciaron la Iglesia en el pasado. Entre los diversos programas prácticos que la IASD ha ideado para cumplir con este propósito, se encuentra el llamado "Culto Joven”, programa que, en esta investigación, se evalúa en las iglesias de Lima de la Asociación Peruana Central Este.

\section{El problema}

Debido a lo descrito, esta investigación plantea el siguiente problema general: ¿Cuál es la situación de los programas del culto joven en la APCE, Lima 2015? Sin embargo, para ser más específicos y efectivos, el problema general ha sido desglosado en los siguientes cuatro problemas específicos:

1. ¿Cuál es la situación de la alabanza en el programa del culto joven en la APCE, Lima 2015?

2. ¿Cuál es la situación de la oración intercesora en el programa del culto joven en la APCE, Lima 2015?

3. ¿Cuál es la situación del testimonio en el programa del culto joven en la APCE, Lima 2015?

4. ¿Cuál es la situación del mensaje en el programa del culto joven en la APCE, Lima 2015?

\section{Objetivos de la investiga- ción}

El objetivo general de la presente investigación es "identificar la situación de los programas del culto joven en la APCE, Lima 2015". El mismo se encuentra desglosado en los siguientes cua-

1. Describir la situación de la alabanza en el programa del culto joven en la APCE, Lima 2015.

2. Describir la situación de la oración intercesora en el programa del culto joven en la APCE, Lima 2015.

3. Describir la situación del testimonio en el programa del culto joven en la APCE, Lima 2015. 
4. Describir la situación del mensaje en el programa del culto joven en la APCE, Lima 2015.

\section{Marcos de la investigación}

Todo quehacer investigativo se construye en un contexto teórico determinado, este contexto, también conocido como marco, permite que la investigación llegue a ser pertinente a la sociedad a la que está dirigida. En este caso, los marcos que rodean esta investigación se desprenden de la historia, de la Biblia y de los escritos de Ellen G. White.

\section{Marco bíblico y teológico del Culto Joven}

El Señor Jesucristo declaró que los que adoran a Dios debieran adorarlo "en espíritu y en verdad; porque también el Padre tales adoradores busca que le adoren". Esto indica que Dios

${ }^{3}$ Juan 4:23. Todas las referencias bíblicas se harán en la versión española Reina-Valera. espera que el adorador puede hacerlo con el pleno conocimiento de la verdad, que es la Palabra de Dios (Juan 17:17), y en el espíritu, es decir, con la actitud de ir a Dios con corazón contrito (Isa 57:15). Otro elemento importante, dentro de la adoración, lo presenta el libro de los Hechos de los Apóstoles, pues une estrechamente la salvación, la adoración y la comunión-xolvavíáa (Hch 2:42, 47).4 En consecuencia, la adoración dentro de la comunidad de creyentes -comunidad que incluye a los jóvenes- se torna en una necesidad indispensable dentro de la experiencia cristiana (Heb 10:25). Ellen G. White al abordar el tema de la salvación, adoración y comunión, afirma lo siguiente:

Aunque Dios no mora en templos hechos por manos humanas, honra con su presencia las asambleas de sus hijos. Prometió que cuando se reuniesen para buscarlo, para reconocer sus pecados y orar unos por

${ }^{4}$ John Nixon, Towards a Theology of Worship: An Application at the Oakwood College Seventh Day Adventist Church (Tesis de Doctorado, Andrews University, 2003), 65. 
otros, él los acompañaría por medio de su Espíritu. Pero, los que se congregan para adorarlo deben desechar todo lo malo. A menos que lo adoren en espíritu y en verdad, así como en la hermosura de la santidad, de nada valdrá que se congreguen. ${ }^{5}$

De este modo, Ellen G. White afirma que una correcta adoración exige que el adorador -adulto, joven o niño- deseche lo malo y se acerque a Dios en espíritu y en verdad.

Asimismo, se debe recordar que de forma explícita, la Biblia expresa una invitación divina al ser humano joven para acordarse de su Creador (Ecl 12:1), invitación que, a lo largo de la historia bíblica, fue aceptada por muchos jóvenes. Dentro de ellos destacan: José, quien rehusó caer en el pecado de la fornicación (Gen 39:9); Josué, quien no se apartaba de en medio del tabernáculo de reunión (Éx 33:11); Samuel, quien ministraba en la presencia de Jehová,

${ }^{5}$ Elena G. de White, Profetas y reyes (Buenos Aires: Asociación Casa Editora Sudamericana, 1990), 35. vestido con un efod de lino (1 Sm 2:18); Daniel, quien rehusó contaminarse con la comida del rey (Dn 1:8), entre otros. Estos fueron jóvenes que decidieron dar gloria y adoración a Dios a través de su diario vivir, apartándose de las pasiones juveniles (2 Tm 2:22) y siendo un ejemplo para los creyentes en palabra, conducta, amor, espíritu, fe y pureza (1 Tm 4:12).

Este contexto hace necesario un culto que pueda invitar a los jóvenes a ser fieles a Dios, y que los desarrolle para llegar a ser la luz del mundo (Mt 5:14-16), convirtiéndolos en ejemplos vivos para otros creyentes y para el mundo entero. Por otro lado, este culto se halla fundamentado en el propósito que el manual de la Iglesia adventista establece, para un servicio o culto dentro de su comunidad:

El propósito de los servicios y de las reuniones de la iglesia es adorar a Dios por su obra creadora y por todos los beneficios de su salvación; entender su Palabra, sus enseñanzas y sus propósitos; confraternizar el uno con el otro en fe y amor; testificar acerca de la fe personal en 
el sacrificio expiatorio de Cristo en la cruz; y aprender cómo cumplir la comisión evangélica y hacer discípulos en todo el mundo (Mat. 28:19, 20). ${ }^{6}$

De manera similar, Ellen G. White brinda algunas directrices para el desarrollo de un culto de adoración que, como se puede notar, deben ser aplicados al culto joven, ella dice:

Nuestras reuniones deben hacerse intensamente interesantes. Deben estar impregnadas por la misma atmósfera del cielo. No oraciones formales simplemente para ocupar el tiempo. Todos deben estar listos para hacer su parte con prontitud y, cuando han cumplido su deber, la reunión debe clausurarse. Así, el interés será mantenido hasta el final. Esto es ofrecer a Dios un culto aceptable. Su servicio debe ser hecho interesante y atrayente, y no [debe] dejarse que degenere en una forma árida.?

${ }^{6}$ Iglesia adventista del séptimo día, Manual de iglesia (Buenos Aires: ACES, 2010), 112.

${ }^{7}$ Elena G de White, Testimonios para la iglesia (Bogotá: Gema Editores, 1998), 5:573.

Poco tiempo después de esta declaración, ella vislumbró algunas escenas de los tiempos finales, en las cuales resaltó la labor de los jóvenes y los niños, así ella declaró:

En las escenas finales, de la historia de esta tierra, muchos de estos niños y jóvenes asombrarán a la gente por su testimonio de la verdad, que darán con sencillez, pero con espíritu y poder. Se les habrá enseñado el temor de Jehová y su corazón habrá sido enternecido por un estudio cuidadoso de la Biblia, acompañado de oración. En el cercano futuro, muchos niños serán dotados del Espíritu de Dios y harán, de la proclamación de la verdad al mundo, una obra que, en aquel entonces, no podrán hacer los miembros adultos. ${ }^{8}$

Y de modo más explícito, al referirse a los jóvenes, en una de sus cartas en el año 1891, dijo: "Nunca, nunca sentí la más mínima perturbación porque el Señor está levantando a la juventud para llevar y transportar cargas más pe-

${ }^{8}$ Elena G. de White, Consejos para maestros, padres y alumnos (Bogotá: Gema Editores, 2005), 158. 
sadas y proclamar el mensaje de la verdad". ${ }^{9}$ Así, ella expone, con seguridad absoluta, que el Señor usa y usará a jóvenes para la proclamación del evangelio. Cabe recordar, referente a ello, que el propio movimiento adventista se formó de la mano de un grupo de jóvenes, cuyo promedio de edad no pasaba los 25 años. Más adelante, en el año 1893, ella declaró:

Las mentes de muchos de los jóvenes son ricas en talentos que permanecen inútiles porque no se les ha dado oportunidad de desarrollarlos...Es preciso que los jóvenes reciban ayudas para el desarrollo; es necesario que se los estimule, se los aliente y se les mueva a la acción. ${ }^{10}$

Pocos días después Ellen G. White, en el Boletín de la Asociación General, declaró:

Tenemos hoy un ejército de jóvenes que puede hacer mucho si es debidamente dirigido y animado. Queremos que nuestros hijos crean la verdad. Queremos que

${ }^{9}$ Elena G. White, Carta 14, 1891.

${ }^{10}$ Elena $G$ de White, Testimonios para la iglesia (Bogotá: Gema Editores, 1998), 4:418. sean bendecidos por Dios. Queremos que participen en planes bien organizados para ayudar a otros jóvenes. Prepárense todos de tal manera que puedan representar debidamente la verdad, dando razón de la esperanza que hay en ellos, y honrando a Dios en cualquier ramo de labor donde estén calificados para actuar. ${ }^{11}$

Finalmente, poco antes de morir, Ellen G. White pidió a la Iglesia para que pueda tomar en consideración la tarea de emplear el talento y las energías de los jóvenes para encauzarlos en la misión, diciendo:

En nuestras iglesias se necesitan los talentos juveniles, bien organizados y preparados. Los jóvenes harán algo con sus rebosantes energías. A menos que estas energías estén encauzadas debidamente, los jóvenes las emplearán de alguna manera que perjudicará su propia espiritualidad, y resultará para daño de aquellos con quienes se asocien. ${ }^{12}$

${ }^{11}$ General Conference Daily Bulletin, 29 de enero 1893.

${ }^{12}$ Elena G. de White, Obreros evangélicos (Buenos Aires: Asociación Casa Editora Sudamericana, 1990), 223. 
Todo lo señalado exige que la Iglesia adventista implemente un plan para los miembros jóvenes de la Iglesia, que los involucre en las actividades de evangelismo y los ayude en su desarrollo espiritual. Por lo tanto, se espera que el Culto Joven sea una respuesta a la situación planteada.

\section{Reseña histórica del Culto Joven en la Iglesia adventista}

La historia del Culto Joven, en la Iglesia adventista, se remonta al año 1879 y al condado de Shiawassee, Michigan, donde Luther Warren, un adolescente de 14 años propuso a su amigo Harry Fenner, un adolescente de 17 años, la formación de una "sociedad de chicos". ${ }^{13}$ Así, al cabo de unos pocos días se reunieron seis a ocho jóvenes en la casa de Luther. El objetivo principal de estas reuniones era ayudar al joven a prepararse para la venida de Cristo Jesús y motivarlo a presentar el evangelio a otros jóvenes, también

\footnotetext{
${ }^{13}$ Luther Warren, "Our First Young People's Society" Youth's Instructor 65, no. 17 (1917): 4.
}

repartían publicaciones de la Iglesia e informaban de las actividades evangelísticas hechas durante los otros días. Aunque, en un inicio, las reuniones que se realizaban en el dormitorio de Warren, solo involucraba a varones y no incluía la participación de adultos, más adelante con el cambio de lugarla sala de la casa-incluyó a damas y a mayores. ${ }^{14}$

Por otra parte, el año 1891, en Antigo Wisconsin, el joven Meade Macguire, viendo las reuniones evangélicas de la "Christian Endeavor Society" y la "Epworth League”, consideró que los jóvenes adventistas necesitaban ese tipo de reuniones. Y aunque, en un primer momento, su idea tuvo una desaprobación general, uno de los líderes mayores lo ayudó y así se organizó la reunión de jóvenes, los días sábados en la noche. Sobre la programación, Macguire declara que consistía "principalmente en cantos, estudio de las Escrituras, mucha oración y testimonio." ${ }^{15}$ Más adelante, poco antes de la

${ }^{14}$ Ibíd., 4.

${ }^{15}$ Meade Macguire, "Early Experiences in Young People's Work". Youth's Instructor 65, no. 17 (1917): 4. 
sesión de la Conferencia General de 1893, A. G. Daniells organizó la "Junior Youth Society" en Adelaide, Australia. Ese mismo año es organizado la "Young People's Society of Christian Service" (Sociedad de Jóvenes del Servicio Cristiano) en College View, Nebraska. Sociedad, cuyo objetivo fue: "asegurar el crecimiento espiritual de los jóvenes, su reclutamiento en todas las actividades misioneras a través de los canales existentes de trabajo, y su elevación a un plano más alto de vida y pensamiento". ${ }^{16}$ El año siguiente, Luther Warren, quien tenía ahora 29 años, organizó la "Sunshine Bands" en el sur de Dakota, todas con prácticas y objetivos similares.

La conferencia de Ohio fue la primera en organizar la sociedad de jóvenes, sociedad que fue reconocida formalmente en el Camp Meeting de 1899. Dicha sociedad llevó por nombre: "Christian Volunteers". Y siguiendo esta iniciativa, el año siguiente, los adventistas de Alemania establecieron una

${ }^{16}$ The Seventh-Day Adventist Encyclopedia (Washington, DC: Review and Herald, 1996), s. v. "Youth Department of Missionary Volunteers". organización juvenil similar. Esto motivó que, el año 1901, el comité de la Asociación General pida al Departamento de Escuela Sabática que se encargue de coordinar las actividades de la sociedad de jóvenes de la IASD. En la sesión de la Asociación General de 1903, la Sra. Plummer reportó un total de 186 sociedades de jóvenes activas, con una membresía total de 3478 jóvenes. ${ }^{17}$ Este puede ser considerado el inicio del actual Culto Joven de la IASD.

El 15 de mayo de 1907, los líderes de la Asociación General reconocieron que el trabajo de los jóvenes había crecido tanto que era necesario el establecimiento de un departamento, de modo que en la reunión de Gland, Suiza, fue creado el "Departamento de Jóvenes" y se invitó a M. E. Kern para dirigirlo. ${ }^{18}$ La primera convención de este departamento, se realizó junto al departamento de Escue-

${ }^{17}$ L. Flora Plummer, "Sabbath School and Young People's Work," General Conference Bulletin 5, no. 8 (1903): 122.

${ }^{18} \mathrm{~W}$. A. Spicer "The General Conference Committee Council at Gland, Switzerland," Review and Herald 84, no. 25 (1907): 4. 
la Sabática del 10 al 21 de julio en Ohio. ${ }^{19}$ M. E. Kern asumió su responsabilidad durante 23 años y desarrolló una variedad de programas que ayudaron a los jóvenes adventistas, de ese entonces, a ser testigos eficientes de Cristo.

Cabe recalcar que hasta 1930 , el énfasis del Departamento de Jóvenes de la IASD fue el reavivamiento personal y la actividad misionera, esto fortaleció a los miembros y les proveyó un fuerte sentido de propósito, estructura y comunidad. ${ }^{20}$ Sin embargo, con el transcurso de los años se fue percibiendo un cambio, la programación del departamento comenzó a ser establecida por adultos y, como consecuencia, la labor de conservación primó en lugar del evangelismo, es decir, se trabajó para

${ }^{19} \mathrm{G}$. B. Thompson, "The Sabbath-School and Young People's Convention," Review and Herald 84, no. 25 (1907): 7.

${ }^{20}$ Cindy Tutsch, "The Development of Adventist Youth Groups and Ellen White's Empowerment of Youth in Evangelism and Service," in Ministering with Millennials: A Report on the $180^{\circ}$ Symposium, ed. Roger L. Dudley and Allan Walshe (Lincoln, NE: AdventSource, 2009), 175. conservar a los miembros jóvenes de la Iglesia, en lugar de trabajar con la juventud adventista por la conversión de otros jóvenes. Por otro lado, la metodología para lograr la salvación de los miembros jóvenes de la iglesia se orientó a entretenerlos y no a organizarlos, con el propósito de proveer oportunidades, donde los propios jóvenes hagan evangelismo sostenido y sistemático. ${ }^{21}$

Frente a esta realidad, en el cuarto trimestre del año 2013, la revista Ação Jovem ${ }^{22}$ publica un voto tomado por la División Sudamericana respecto al Departamento del Ministerio Joven. Bajo el título "Nova ação joven”, el Pr. Areli Barbosa, director del Ministerio Joven de la División Sudamericana, comunica el voto, texto que reza de la siguiente manera:

\section{Considerando que:}

1. Los jóvenes necesitan tener un culto que retrate su

${ }^{21}$ Ibid.

${ }^{22}$ "Voto para el culto Joven - DSA" http://www.adventistas.org/pt/jovens/2014/02/28/voto-para-culto-jovem-dsa/, (Consultado: 6 de enero 2016). 
realidad, cuyo contenido puede ayudarles en sus luchas y desafíos.

2. Que muchas actividades como el trabajo misionero, las comisiones y las reuniones administrativas, que cruzan en horario con el culto joven, lo han debilitado.

3. La importancia de reafirmar un día y hora para fortalecer la programación.

4. El reto de contar con la presencia de líderes de las iglesias locales en el Culto Joven.

5. La iniciativa para fortalecer el culto de jóvenes precisa de un compromiso de todos los líderes de la Iglesia.

6. El formato actual del Culto Joven no logra atraer a la mayoría de los jóvenes.

7. Este culto ha tenido diferentes nombres: Misioneros Voluntarios (MV), la Liga de los Jóvenes, Jóvenes Adventistas (JA), lo cual revela los diversos intentos de adaptar el lenguaje y formato del programa adventista para jóvenes.

8. Las redes sociales han promovido cambios importantes en la conducta social de los jóvenes.

Se propone que:

1. Se reafirme el Culto Joven como un programa importante para la vida espiritual de la Iglesia.

2. El Culto Joven se dé en el sábado de tarde, cuando no haya cruce con otras actividades de la Iglesia.

3. El lanzamiento del nuevo formato del Culto Joven se dé el día 8 de febrero del 2014, por el canal ejecutivo con transmisión vía satélite.

4. En el año 2014 haya capacitación para los directores del Ministerio Joven de la iglesia local, a través de cursos y encuentros de liderazgo.

5. El liderazgo de la iglesia local y su pastor estén presentes en el Culto Joven. 
6. El formato de Culto Joven priorice cuatro elementos: Alabanza, Oración de intercesión, Testimonio y Mensaje.

7. Culto Joven sea el nombre de este programa.

8. La revista Acción Joven y otros materiales promuevan este nuevo formato, ayudando al director local para adaptarse a las nuevas necesidades.

9. Las redes sociales potencialicen la difusión y incentiven el debate anticipado de los temas de cada sábado.

Debido a ello, a partir del año 2014, se ha ido implementando y fortaleciendo este plan, muchas de las iglesias adventistas de la APCE lo están poniendo en práctica y, como se ha dicho, la presente investigación es una especie de evaluación a dicho plan.

\section{Antecedentes}

Como se pudo ver, el programa llamado "Culto Joven" es bastante reciente, debido a ello, se podría decir que esta investigación es pionera en lo que respecta a este programa. Sin embargo, una investigación afín fue realizada por el Dr. Felipe Esteban Silva, publicada bajo el título "El 'culto joven' y su relación con el compromiso cristiano de los jóvenes de las iglesias adventistas del séptimo día de España y Santa Isabel de Lima Metropolitana, año 2009". ${ }^{23}$ Aunque la investigación del Dr. Esteban tiene objetivos diferentes y evalúa un programa y variables diferentes a las que se evalúa en la presente investigación, algunas conclusiones obtenidas por el Dr. Esteban pertinentes al tema fueron:

1. La relación directa entre el programa, el ambiente físico del culto joven y el compromiso cristiano.

2. Demostrar que los jóvenes que participan, en el culto

${ }^{23}$ Felipe Esteban Silva, "El 'culto joven' y su relación con el compromiso cristiano de los jóvenes de las iglesias adventistas del séptimo día de España y Santa Isabel de Lima Metropolitana, año 2009," Estrategias para el cumplimiento de la misión 10, no. 2 (2013): 26-56. 
joven, tienen mayor participación activa en el programa de Escuela Sabática y en las reuniones de grupos pequeños, que usan más la Biblia y el folleto de Escuela Sabática, que son más puntuales y tienen mayor conocimiento de las doctrinas, que entienden mejor el mensaje bíblico y satisfacen mejor sus necesidades espirituales.

Por lo tanto, se puede afirmar que la investigación del Dr. Esteban apoya la creación del programa llamado "Culto joven", pues como se nota, esa investigación encontró que existe correlación positiva significativa entre el compromiso cristiano y el culto joven. Y aun cuando el "culto joven", que el Dr. Esteban investigó, no es el programa que el presente artículo aborda, se puede afirmar que la realización de un programa que enfatice áreas que imiten al culto del sábado en la mañana, contribuye, de forma significativa, a elevar el compromiso cristiano en los jóvenes de la IASD.

\section{Modelo de Culto Joven}

Como se sabe un modelo es una forma de concebir la práctica de los procesos en una determinada institución o programa. En el caso de una institución religiosa, comprende procesos relativos a cuestiones pedagógicas religiosascómo se aprende la verdad y cómo se la enseña- también comprende las metodologías más adecuadas para la asimilación significativa de los conocimientos bíblicos, el cómo se desarrolla las habilidades y valores cristianos, las consideraciones epistemológicas entorno a la teología, las aplicaciones prácticas de ella, el currículo -en este caso el preparado para aquellos que participan del culto joven-y la evaluación de los objetivos del culto joven. ${ }^{24}$

Hacer todo ello, no es el objetivo de esta investigación, sin embargo, se debe reconocer que la implementación del culto joven, dividido en cuatro partes, eco de los programas ideados por

${ }^{24}$ Grupo pedagógico de la Universidad Mariana, Modelo pedagógico (San Juan de Pasto: Editorial Universidad Mariana, 2008), 1-142. 
Macguire, podría ser la base sobre la cual se establezca este modelo ausente, estas cuatro partes, las que se ha evaluado en esta investigación, a saber son: alabanza, oración, testimonio y mensaje. No obstante, luego de afirmar la inexistencia de un modelo de culto joven en la Iglesia adventista, se hacen algunas propuestas que pueden ser de gran ayuda para la creación de un Modelo de Culto Joven Adventista, en el futuro.

\section{Pedagogía para el joven del siglo XXI}

El tiempo y momento que nos toca vivir, no fue escogido ni puede ser seleccionado por nosotros, el siglo XXI, como muchos pedagogos lo han manifestado, exige un cambio en la forma que se enseña, pues la forma en la que se aprende ha cambiado. ${ }^{25}$ En este sentido, este artículo motiva a quienes compete crear un sistema pedagógico que sea efectivo y

${ }^{25}$ Jordi Riera i Romaní y M. Civís I. Zaragoza, "La pedagogía profesional del siglo XXI," Educación 21, no. 11 (2008): 133-154; Viviane Castro Camozzato, "Pedagogias do Presente" Educação \& Realidade 39, no. 2 (2014): 573-593. acorde con la forma en la que hoy aprenden los jóvenes, los cuales hacen parte del culto joven.

Al parecer hoy en día lo jóvenes aprenden muy poco en los cultos o reuniones con índole formativa, existen medios educativos más poderosos, que en "silencio"-sin llamarse profesor, pastor o culto- hacen una labor educativa poderosa. Ejemplo de ello es la $\mathrm{TV}$, respecto a ella el profesor y comunicador Nicholas Johnson dijo: "Toda la televisión es televisión educativa. La pregunta es: ¿qué es lo que enseña?". ${ }^{26}$ Por lo señalado, se cree que la elaboración de un modelo de culto joven, debe tomar en cuenta la enseñanza invisible, ${ }^{27}$ y debido a ello, el perfil requerido para los líderes de jóvenes debe incluir, además

${ }^{26}$ "El nautilus tendencia en TV: 20.000 lenguas a través de la pantalla".https://elnautilus.wordpress. com/2008/10/29/las-mejores-frasesacerca-de-la-tele/, (Consultado el 6 de Enero de 2105).

${ }^{27}$ Para más información sobre el tema véase Cristóbal Cobo Romaní y John W. Moravec, Aprendizaje invisible: hacia una nueva ecología de la educación (Barcelona: Edicions de la Universitat de Barcelona, 2011). 
de aspectos bíblicos y espirituales, asuntos tecnológicos. Pues, como lo dijo George Siemens, la enseñanza actual tiene que ver con conectar al educando con el mundo, ${ }^{28}$ que, en este caso, debe ser el gran mundo al cual pertenece todo aquel que asiste al culto joven.

Por otro lado, la elaboración de un modelo de culto joven debe tomar en cuenta lo defendido por Roger Schank, el aprendizaje natural, pues como él señala existe una brecha insalvable entre el aprendizaje teórico y el aprendizaje práctico. ${ }^{29} \mathrm{La}$ pedagogía en el culto joven, por lo tanto, debe incluir elementos prácticos y no solo teóricos.

${ }^{28}$ George Siemens, "Conectivismo - Lima, 2012," Exposición en el Encuentro Internacional de Educación, 2012 2013 en Lima, Perú, bajo https://www. youtube.com/watch? $\mathrm{v}=$ s77NwWkVth8, (consultado: 18 de octubre, 2015).

${ }^{29}$ Roger Schank, "El aprendizaje no ha cambiado, entonces ¿por qué debería cambiar la enseñanza?" Exposición en el VII Encuentro Internacional de Educación, 2012 - 2013 en ciudad de México, bajo https://www.youtube.com/ watch? $\mathrm{v}=\mathrm{kIquall} 9 \mathrm{HjM}$ (consultado: 18 de octubre, 2015).

\section{Metodología del Culto Joven}

La metodología empleada para el culto joven debe ser apropiada y efectiva, y aun cuando no es recomendable que sea rígida, sino flexible, para adaptarse a la situación de los jóvenes en su contexto particular, ella también debería ser sólida y enfocada a cumplir el "objetivo último de desarrollar el carácter, la buena ciudadanía y una vida cristiana auténtica", ${ }^{30}$ lo que implica una vida de adoración, comunión y misión.

No se debe confundir el método pedagógico con la metodología a emplear en un culto joven. Relacionado con esto se encuentra la metodología de manejo de grupos, se recomienda, que como parte del modelo de culto joven, se determine la cantidad de personas que pueden ser parte de él. Pero si el número es mayor del recomendado, el modelo debe presentar

${ }^{30}$ Edward M. Cadwallader, Principios de la educación adventista en el pensamiento de Elena G. de White: filosofía, objetivos, métodos y misión (Lima: Ediciones Universidad Peruana Unión, 2010), 393. 
una solución a dicho problema. La observación, los proyectos y otros aspectos que hagan parte del culto joven, se deben tomar en cuenta cuando se elabore el modelo de culto joven.

\section{Desarrollo de habilida- des y valores en el Culto Joven}

La Iglesia adventista tiene como principio el desarrollo integral de la persona, sin embargo, se debe reconocer que existen mejores momentos en la vida de una persona para alcanzar este desarrollo integral. Por lo tanto, el modelo de culto joven debe incluir actividades, programas y sistemas que permitan un desarrollo natural de habilidades y valores en los jóvenes.

Sin embargo, es también necesario que se determinen los valores y habilidades que se enfatizará en el culto joven. Hoy en día existen una gran cantidad de valores y habilidades que podrían encontrarse en riña con los principios bíblicos que deben gobernar la elaboración del modelo de culto joven.
Debido a ello se deben diseñar situaciones, procesos y estrategias cognitivas y prácticas, fundamentadas en los principios bíblicos, que permitan que el culto joven alcance su objetivo propuesto.

\section{Teología bíblica relacio- nada al Culto Joven}

Esta sección, en el modelo de culto joven, debe incluir las consideraciones epistemológicas del culto joven y las aplicaciones prácticas de ella. Algo de ello se ha dicho ya en el marco bíblico y teológico de esta investigación (2.1), sin embargo, se reconoce que preguntas importantes como ipor qué se debe hacer un programa especial para los jóvenes? Debe ser respondido desde una plataforma bíblica.

En el contexto adventista, también se debería tomar en cuenta las publicaciones hechas por Ellen G. White, sobre adoración, servicio y misión y relacionadas a la juventud, ella en uno de sus escritos declara:

Las iglesias de diferentes localidades deben sentir que descansa sobre ellas la solemne responsa- 
bilidad de educar a los jóvenes y preparar sus talentos para que se dediquen a la obra misionera. Cuando ellos vean en la iglesia [jóvenes que] prometen llegar a ser obreros útiles, pero que no pueden sostenerse en la escuela, deben asumir la responsabilidad de mandarlos a una de nuestras escuelas. ${ }^{31}$

\section{El currículo del Culto Joven}

El currículo preparado para aquellos que participan del culto joven, no debe incluir únicamente el currículo expreso, formal $\mathrm{u}$ oficial, aquel que presenta los alcances, secuencias, sílabos, contenidos y objetivos. ${ }^{32}$ Sino también debe incluir el currículo oculto, aquel que aun cuando no es reconocido de forma explícita, muchas veces produce un mayor impacto que el currículo oficial. ${ }^{33}$ Este cu-

${ }^{31}$ Ellen G. White, Consejos para los Maestros (Mountain View, CA: Pacific Press Publishing Association, 1971), 67.

${ }^{32}$ Luis A. Malagón Plata, Currículo y pertinencia en la educación superior, ed. (Bogotá: Cooperativa Editorial Magisterio, 2007), 77-122.

${ }^{33}$ María Teresa Pérez García, Profesión, docente de medicina: ¿Se puede rrículo se relaciona con temas afines al género, hábitos, autoridad, raza, valores, presuposiciones religiosas, etc.

Otro currículo que debe ser tomado en cuenta, es el operacional, el mismo que debe organizarse de forma gradual y escalonada. Es decir allí debe presentarse lo que se enseñará, durante los trimestres, años y quinquenios, como también los resultados que se espera tener como consecuencia de esa enseñanza. En esto el modelo, elaborado por el Ministerio del Menor de la IASD, denominado Eslabones de la Gracia, debería servir imitado, allí se contempla un currículo para 12 años, aplicado a niños de 0 a 14 años de edad, en el que se enseñan 624 lecciones. ${ }^{34}$ Finalmente, no se de-

conjugar en un solo profesional el ejercicio de las dos profesiones? (Bogotá: Universidad Nacional de Colombia, 2002), 41.

${ }^{34}$ Departamento de los Ministerios del Niño de la Asociación General de los Adventistas del Séptimo Día, Manual de los ministerios del niño: una guía, paso a paso, para líderes de niños alrededor del mundo. http:// www.iglesiaadventistaagape.org/Documents/Manual\%20de\%20los\%20 Ministerios\%20del\%20Ni\%C3\%B1o. 
bería dejar de lado el currículo nulo, es decir, los temas que no serán enseñados, centrándose en las razones por las cuales se obviará dicho contenido.

\section{Evaluación del programa de Culto Joven}

Finalmente, un modelo de culto joven debe incluir un sistema de evaluación permanente, para cada etapa del modelo y para la totalidad del mismo. Este sistema de evaluación debe centrarse en los objetivos del modelo, en sus diferentes etapas.

\section{Análisis de los resultados}

La encuesta aplicada fue respondida acorde a las indicaciones por 428 miembros de 70 iglesias de la Asociación Peruana Central Este de la ciudad de Lima. Se debe aclarar que para hacer, de esta investigación, un trabajo más objetivo, se aplicó la encuesta por la tarde del sábado, es decir, solo a los miembros que generalmente asisten al culto joven. A continua-

pdf, (Consultado el 6 de Enero de 2016). ción se presenta los resultados obtenidos y, en base a ello, se expone el análisis a seis áreas que están directamente relacionadas con el programa denominado culto joven.

\section{Sobre los datos generales}

La tabla 1 muestra que 114 encuestados oscilan entre los $15 \mathrm{y}$ 24 años de edad, lo que representa el 26,6\% del total. 93 personas se encuentran entre las edades de 25 a 34 años, lo que representa el $21,7 \%$ de los encuestados. Sumados estos dos grupos hacen un total de 207 miembros de iglesia encuestados, por lo tanto, las personas entre 15 y 34 años son el grupo que más frecuentan el culto joven.

Por otro lado, de los encuestados, 184 son varones y 244 mujeres, que representan al $43 \%$ y $57 \%$ respectivamente. 252 de ellos son solteros y 157 son casados, es decir $58,9 \%$ son solteros y $36,7 \%$ son casados. Sin embargo, también existen, divorciados, viudos y convivientes, quienes representan el $0.9 \%, 2,3 \%$ y $1.2 \%$ respectivamente, del total de encuestados. 
En cuanto al nivel educativo, 174 personas tienen educación superior $(40 \%), 159$ tienen educación secundaria completa $(37,1 \%)$ y 95 de ellos tienen primaria completa $(22,2 \%)$. Por otro lado, del total de encuestados, 405 son miembros bautizados de la Iglesia Adventista del Séptimo Día y 23 no lo son, cantidades que en porcentajes equivalen al $94,6 \%$ y $5,4 \%$, respectivamente.

Referente a la asistencia al "culto joven", 305 personas afirman que asisten 4 veces al mes (71,3\%), 60 encuestados indican que asisten 3 veces al mes (14\%), 33 lo hacen 2 veces por mes $(7,7 \%)$ y 30 asisten al culto joven solamente 1 vez por mes (7\%).

Tabla $\mathrm{N}^{\circ} 1$ Datos generales

\begin{tabular}{|ll|l|l|}
\hline & & Frecuencia & Porcentaje \\
\hline \multirow{5}{*}{ Edad } & Menores de 14 años & 47 & 11.0 \\
& Entre 15 y 24 años & 114 & 26.6 \\
& Entre 25 y 34 años & 93 & 21.7 \\
& Entre 35 y 44 años & 61 & 14.3 \\
& Entre 45 y 54 años & 65 & 15.2 \\
& Mayores de 55 años & 48 & 11.2 \\
& Total & 428 & 100.0 \\
\hline \multirow{5}{*}{ Sexo } & Masculino & 184 & 43.0 \\
& Femenino & 244 & 57.0 \\
& Total & 428 & 100.0 \\
\hline \multirow{5}{*}{ Estado civil } & Soltero & 252 & 58.9 \\
& Casado & 157 & 36.7 \\
& Divorciado & 4 & .9 \\
& Viudo & 10 & 2.3 \\
& Conviviente & 5 & 1.2 \\
Nivel educativo & Total & 428 & 100.0 \\
& Primaria & 95 & 22.2 \\
& Secundara & 159 & 37.1 \\
& Superior & 174 & 40.7 \\
& Total & 428 & 100.0 \\
\hline
\end{tabular}




\begin{tabular}{|ll|l|l|}
\hline \multirow{3}{*}{ Miembro bautizado } & Sí & 405 & 94.6 \\
& No & 23 & 5.4 \\
& Total & 428 & 100.0 \\
\hline & 1 vez al mes & 30 & 7.0 \\
N.․ de sábados que & 2 veces al mes & 33 & 7.7 \\
asiste & 3 veces al mes & 60 & 14.0 \\
& 4 veces al mes & 305 & 71.3 \\
& Total & 428 & 100.0 \\
\hline
\end{tabular}

\section{Sobre feligresía total y asistencia al Culto Joven}

La tabla 2 expone el promedio de asistencia al Culto Joven en las iglesias de la APCE, zona de Lima, en relación con la cantidad total de feligreses de la iglesia. En iglesias que tienen menos de 50 miembros, asisten un promedio de 21 personas (54,6\%); en iglesias que tienen entre 50 a 100 miembros, son 30 los asistentes
(42,3\%). Asimismo, son 43 los que asisten al programa del culto joven en iglesias que tienen entre 100 a 150 miembros (37,8\%), de igual forma, 56 personas asisten al programa referido en iglesias que tienen entre 150 a 200 miembros $(29,4 \%)$, y en iglesias que tienen más de 200 miembros, la asistencia al programa del culto joven disminuye a 43 personas, cantidad que representa solo al 14,8\% de la totalidad de su feligresía.

Tabla $\mathrm{N}^{\circ} 2$ Promedio de asistencia al Culto Joven

\begin{tabular}{|l|c|c|}
\hline & Promedio de asistencia & \% de miembros \\
\hline Iglesias con menos de 50 miembros & 21.0 & 54.6 \\
\hline Iglesias con 50 a 100 miembros & 30.0 & 42.3 \\
\hline Iglesias con 100 a 150 miembros & 43.0 & 37.8 \\
\hline Iglesias con 150 a 200 miembros & 56.0 & 29.4 \\
\hline Iglesias con más de 200 miembros & 43.0 & 14.8 \\
\hline
\end{tabular}

\section{Sobre la alabanza en el Culto Joven}

La tabla 3 muestra que 214 personas creen que el director de alabanzas del Culto Joven es permanente, es decir, no cambia; los otros 214 encuestados, por su parte, creen que cambia permanentemente, por lo tanto, existe una 
representación del 50\% para cada una de las respuestas. En relación al buen testimonio del director de alabanzas, dentro y fuera de la iglesia, 287 personas contestaron que tiene un buen testimonio $(67,1 \%)$ y 141 respondieron que no $(32,9 \%)$. En cuanto a si existe un conjunto musical integrado por jóvenes encargados de dirigir las alabanzas en el programa, 140 encuestados respondieron que sí, mientras que 288 contestaron que no, por lo tanto, el 32,7\% declaró que sí y el 67,3\% dijo que no.

Respecto a si las partes especiales son evaluadas antes de ser presentadas en el Culto Joven, 186 contestaron que sí (43.5\%), mientras que 242 encuestados contestaron que no $(56,5 \%)$. También se encontró que 227 (53\%) personas respondieron que en los programas del culto joven sí se aprenden nuevos himnos y 201 (47\%) dijeron que no. Asimismo, del total de las personas encuestadas, 294 $(68,7 \%)$ afirmaron que las alabanzas del culto joven tienen relación con el tema central del programa, mientras que 134 (34\%) personas dijeron que no.

Tabla N³ Alabanza en el Culto Joven

\begin{tabular}{|c|c|c|c|}
\hline & & Frecuencia & Porcentaje \\
\hline \multirow{2}{*}{ El director de alabanzas es permanente } & Sí & 214 & 50.0 \\
\hline & No & 214 & 50.0 \\
\hline \multirow{2}{*}{ El director de alabanzas tiene buen testimonio } & Sí & 287 & 67.1 \\
\hline & $\mathrm{No}$ & 141 & 32.9 \\
\hline \multirow{2}{*}{ Un conjunto musical dirige las alabanzas } & Sí & 140 & 32.7 \\
\hline & No & 288 & 67.3 \\
\hline \multirow{2}{*}{ Los números especiales se evalúan antes de presentarse } & Sí & 186 & 43.5 \\
\hline & No & 242 & 56.5 \\
\hline \multirow{2}{*}{ Se aprenden nuevos himnos en el Culto Joven } & Sí & 227 & 53.0 \\
\hline & No & 201 & 47.0 \\
\hline \multirow{3}{*}{$\begin{array}{l}\text { Las alabanzas tienen relación con el tema central del } \\
\text { Culto Joven }\end{array}$} & Sí & 294 & 68.7 \\
\hline & No & 134 & 31.3 \\
\hline & Total & 428 & 100.0 \\
\hline
\end{tabular}




\section{Sobre la oración interce- sora en el Culto Joven}

En la tabla 4 se observa que 387 (90.4\%) personas afirman que en el programa del Culto Joven existe un espacio dedicado a la oración intercesora, mientras que $41(9,6 \%)$ encuestados respondieron que no. $\mathrm{Al}$ mismo tiempo, la tabla muestra que 367 (85.7\%) miembros de iglesia perciben que el espacio de la oración intercesora es inspiradora, mientras que $61(14.3 \%)$ de ellos contestaron que no. De otro lado, sobre la existencia de un registro para pedidos y oraciones contestadas, $230(53.7 \%)$ contestaron positivamente, mientras que 198 $(46,3 \%)$ dijeron que no. Respecto a la pregunta si la oración intercesora tiene relación con el tema central del programa del Culto Joven, 209 respondieron que sí, lo cual significa el $48,8 \%$ de los encuestados, mientras que $219 \mathrm{di}$ jeron que no, es decir el 51,2\% de la población encuestada.

Tabla $N^{\circ} 4$ Oración Intercesora

\begin{tabular}{|l|c|c|c|}
\hline & & Frecuencia & Porcentaje \\
\hline \multirow{2}{*}{ Se realiza la oración intercesora en el Culto Joven } & Sí & 387 & 90.4 \\
\cline { 2 - 4 } & No & 41 & 9.6 \\
\hline \multirow{2}{*}{ El espacio de oración intercesora es inspiradora } & Sí & 367 & 85.7 \\
\cline { 2 - 4 } & No & 61 & 14.3 \\
\hline \multirow{2}{*}{ Existe un registro de pedidos y oraciones contestadas } & Sí & 230 & 53.7 \\
\cline { 2 - 4 } & No & 198 & 46.3 \\
\hline \multirow{2}{*}{ La oración intercesora tiene relación con el tema central } & Sí & 209 & 48.8 \\
\cline { 2 - 4 } & No & 219 & 51.2 \\
\hline & Total & 428 & 100.0 \\
\hline
\end{tabular}




\section{Sobre los testimonios en el Culto Joven}

La tabla 5 evidencia que 203 $(47,4 \%)$ miembros de iglesia respondieron que los testimonios expuestos en el culto joven son congruentes con el tema central, mientras que los que opinan que no suman la cantidad de 225 $(52,6 \%)$. A la pregunta, si se emplea vídeos y/o fotos durante la narración de los testimonios, 121 $(28,3 \%)$ personas consideraron que sí, mientras que 307 (71\%) dijeron que no. Por otro lado, sobre la pregunta, si los testimonios son inspiradores, $321(75 \%)$ contestaron que sí, mientras que 107
(25\%) respondieron que no. En relación al número de veces que se presentan por mes los testimonios en el culto joven, 115 (26.9\%) respondieron 1 vez al mes, 119 (27.8\%) dijeron 2 veces, $30(7 \%)$ contestaron 3 veces, 138 (32.2\%) refirieron que 4 veces al mes, mientras que $26(6.1 \%)$ dijeron que nunca se presentan testimonios en el programa del culto joven de su iglesia. Ante la pregunta sobre el tiempo que se dedica al espacio de los testimonios en el programa del culto joven, 118 (27.6\%) dijeron que 5 minutos, $182(42.5 \%)$ contestaron que 10 minutos, 63 (14.7\%) respondieron que $15 \mathrm{mi}$ nutos y $65(15.2 \%)$ marcaron la opción de 20 minutos.

Tabla $\mathrm{N}^{\circ} 5$ Testimonios

\begin{tabular}{|l|c|c|c|}
\hline & & Frecuencia & Porcentaje \\
\hline \multirow{2}{*}{$\begin{array}{l}\text { Los testimonios son congruentes con el tema cen- } \\
\text { tral }\end{array}$} & Sí & 203 & 47.4 \\
\cline { 2 - 4 } $\begin{array}{l}\text { Se emplea fotos y/o vídeos durante la narración del } \\
\text { testimonio }\end{array}$ & No & 225 & 52.6 \\
\hline \multirow{2}{*}{ Los testimonios son inspiradores } & No & 121 & 28.3 \\
\hline & Sí & 307 & 71.7 \\
\cline { 2 - 4 } & No & 107 & 75.0 \\
\hline & Total & 428 & 25.0 \\
\hline \multirow{3}{*}{$\begin{array}{l}\text { N. }{ }^{\circ} \text { de veces al mes que se presentan testimonios en } \\
\text { el Culto Joven }\end{array}$} & 1 & 115 & 260.0 \\
\cline { 2 - 4 } & 2 & 119 & 27.8 \\
\cline { 2 - 4 } & 4 & 30 & 7.0 \\
\cline { 2 - 4 } & Nunca & 138 & 32.2 \\
\cline { 2 - 4 } & Total & 428 & 6.1 \\
\hline
\end{tabular}




\begin{tabular}{|l|c|c|c|}
\hline \multirow{4}{*}{$\begin{array}{l}\text { Minutos que se dedican al testimonio en el Culto } \\
\text { Joven }\end{array}$} & 5 & 118 & 27.6 \\
\cline { 2 - 4 } & 10 & 182 & 42.5 \\
\cline { 2 - 4 } & 15 & 63 & 14.7 \\
\cline { 2 - 4 } & 20 & 65 & 15.2 \\
\cline { 2 - 4 } & Total & 428 & 100.0 \\
\hline
\end{tabular}

\section{Sobre el mensaje en el Culto Joven}

En la tabla 6 se observa que $378(88,3 \%)$ encuestados refieren que durante la exposición del mensaje se usa la Biblia, mientras que $50(11,7 \%)$ de ellos contestaron que no. A la pregunta si se lee los escritos de Elena G. de White en la exposición del mensaje, 240 $(56,1 \%)$ indicaron que sí, mientras que $188(43,9 \%)$ dijeron que no. Al mismo tiempo, 349 (81,5\%) personas contestaron que los mensajes del culto joven son inspiradores, mientras que 79 (18,5\%) de ellos refirieron que no. Sobre la preparación anticipada de los mensajes, 331 (77\%) contestaron que se nota preparación anticipada, mientras que 97 (22,7\%) dijeron que no. En cuanto a la variedad de predicadores por mes, 358 $(83,6 \%)$ contestaron que existen diferentes predicadores, mientras que $70(16,4 \%)$ opinaron lo con- trario. A la pregunta si los mensajes predicados, en el programa del culto joven, son aplicables a la realidad de la problemática de la iglesia, $353(82,5 \%)$ contestaron que sí, mientras que 75 (17,5\%) dijeron que no. Por otro lado, respecto a la realización de programas paralelos al programa del culto joven, $161(37,6 \%)$ contestaron que sí, 267 (62,4\%) refirieron que no. En cuanto al número de veces que por mes se presenta el mensaje en el culto joven, 72 (16,8\%) encuestados dijeron que una vez, $39(9.1 \%)$ opinaron que dos veces, 25 (5.8\%) de ellos refirieron que tres veces y $292(68,2 \%)$ indicaron que cuatro veces. A la pregunta, cuantos minutos se dedica al mensaje del culto joven, $73(17.1 \%)$ dijeron que $10 \mathrm{minu}$ tos, $67(15.7 \%)$ indicaron que 15 , $108(25.2 \%)$ refirieron que 20 minutos, 63 (14.7\%) dijeron que 25 minutos, 82 (19.2\%) personas dijeron que 30 minutos, mientras que $35(8.2 \%)$ de ellas señalaron 
que 35 minutos. Finalmente, 237 $(55,4 \%)$ personas contestaron que el pastor predica en el culto joven una vez al mes, 50 (11,7\%) dijeron que el pastor predica 2 veces por mes, $9(2,1 \%)$ personas contesta- ron que el pastor predica 3 veces al mes, 30 (7\%) de ellos opinaron que el pastor predica 4 veces al mes, y $102(23,8 \%)$ refirieron que el pastor nunca predica en el culto joven de su iglesia.

Tabla $\mathrm{N}^{\circ} 6$ Mensaje

\begin{tabular}{|c|c|c|c|}
\hline & & Frecuencia & Porcentaje \\
\hline \multirow{2}{*}{ Se lee la Biblia durante el mensaje del Culto Joven } & Sí & 378 & 88.3 \\
\hline & No & 50 & 11.7 \\
\hline \multirow{2}{*}{$\begin{array}{l}\text { Se lee los escritos de Elena G. de White durante el mensa- } \\
\text { je del Culto Joven }\end{array}$} & Sí & 240 & 56.1 \\
\hline & No & 188 & 43.9 \\
\hline \multirow{2}{*}{ Los mensajes son inspiradores } & Sí & 349 & 81.5 \\
\hline & No & 79 & 18.5 \\
\hline \multirow{2}{*}{ Se percibe que el mensaje fue preparado con anticipación } & Sí & 331 & 77.3 \\
\hline & No & 97 & 22.7 \\
\hline \multirow{2}{*}{ Durante un mes, los predicadores son diferentes } & Sí & 358 & 83.6 \\
\hline & No & 70 & 16.4 \\
\hline \multirow{2}{*}{ Los mensajes son aplicables a la realidad de la iglesia } & Sí & 353 & 82.5 \\
\hline & No & 75 & 17.5 \\
\hline \multirow{2}{*}{$\begin{array}{l}\text { Se realizan otras reuniones paralelas al programa del } \\
\text { Culto Joven }\end{array}$} & Sí & 161 & 37.6 \\
\hline & No & 267 & 62.4 \\
\hline \multirow{5}{*}{$\begin{array}{l}\text { N. }{ }^{\circ} \text { de veces al mes que se presenta el mensaje en el Culto } \\
\text { Joven }\end{array}$} & $1 \mathrm{vez}$ & 72 & 16.8 \\
\hline & 2 veces & 39 & 9.1 \\
\hline & 3 veces & 25 & 5.8 \\
\hline & 4 veces & 292 & 68.2 \\
\hline & Total & 428 & 100.0 \\
\hline \multirow{7}{*}{ Minutos que se dedica al mensaje del Culto Joven } & $10 \mathrm{~min}$ & 73 & 17.1 \\
\hline & $15 \mathrm{~min}$ & 67 & 15.7 \\
\hline & $20 \mathrm{~min}$ & 108 & 25.2 \\
\hline & $25 \mathrm{~min}$ & 63 & 14.7 \\
\hline & $30 \mathrm{~min}$ & 82 & 19.2 \\
\hline & $35 \mathrm{~min}$ & 35 & 8.2 \\
\hline & Total & 428 & 100.0 \\
\hline \multirow{5}{*}{$\begin{array}{l}\text { N. }{ }^{\circ} \text { de veces al mes que el pastor predica el mensaje en el } \\
\text { Culto Joven }\end{array}$} & $1 \mathrm{vez}$ & 237 & 55.4 \\
\hline & 2 veces & 50 & 11.7 \\
\hline & 3 veces & 9 & 2.1 \\
\hline & 4 veces & 30 & 7.0 \\
\hline & Nunca & 102 & 23.8 \\
\hline \multicolumn{2}{|l|}{ Total } & 428 & 100.0 \\
\hline
\end{tabular}




\section{Discusiones}

En el presente estudio se determinó que el más alto porcentaje de los encuestados son solteros con educación superior y del nivel secundario quienes, en su mayoría, son mujeres y se encuentran entre las edades de 15 a 34 años de edad. Esta mayoría de encuestados asisten todos los sábados del mes a los programas del culto joven y, de preferencia, lo hacen a las iglesia más pequeñas porque el resultado refleja que las iglesias más grandes son las menos concurridas en el mencionado programa.

En cuanto al espacio de alabanzas, dentro del programa del culto joven, se destaca que el director de cantos refleja un buen testimonio y que su particiación es permanente; además la mayor parte de la población encuestada indica que no existe un conjunto musical que dirija este espacio en el culto joven. También el estudio determinó que, en su mayoría, las partes especiales no se evalúan antes de su presentación, pero que sí se aprenden nuevos himnos, finalmente sobre este aspecto se determinó que generalmente las alabanzas tienen relación con el tema central de culto joven.

En relación a la oración intercesora, en el programa del culto joven, el estudio determinó que sí existe, además el mayor porcentaje de los encuestados afirmó que este espacio es inspirador y casi la mitad de ellos dio fe de que existe un registro de pedidos y oraciones contestadas en sus iglesias; además refirieron ellos que la oración intercesora tiene relación con el tema central del culto joven.

Por otro lado, la investigación encontró que el mayor porcentaje de encuestados dice que los testimonios expuestos en el programa del culto joven son congruentes con el tema central, sin embargo, también se dijo que para este espacio se emplea muy pocas fotos 0 vídeos. Además, la mayoría de los miembros de iglesia encuestados indicaron que los testimonios duran un tiempo aproximado de 10 minutos y que son inspiradores.

El estudio también halló que mayormente el mensaje central, expuesto en el culto joven, dura entre 20 a 30 minutos y que es inspirador, lo que al parecer se re- 
laciona con la lectura de la Biblia y los escritos de Elena G. de White. Por otro lado, el mayor porcentaje de la población encuestada percibe que el mensaje fue preparado con anticipación y que estos son aplicables a la realidad de la iglesia. Sin embargo, también se puede afirmar que las iglesias tienen reuniones o programas paralelos al culto joven, lo cual distrae o disminuye la importancia que tiene el mencionado programa. A esta preocupación se suma la escasa o nula participación del pastor de iglesia, como predicador del mensaje central, en los programas del culto joven.

A pesar de que la DSA sugirió un nuevo lanzamiento del culto joven, se encontraron los siguientes desafíos que todavía no se han corregido:

1. Solo el $50 \%$ de iglesias tiene un director de cantos permanente.

2. Un tercio menciona que el director de cantos no tiene buen testimonio.

3. Un tercio menciona que existe un conjunto musical.
4. La mitad de la iglesia menciona que la oración intercesora no tiene relación con el tema central.

5. Casi el 50\% de la iglesia dice que el testimonio no tiene relación con el tema central.

6. Un cuarto de iglesia dice que los testimonios no son inspiradores.

7. Un tercio de iglesias dice que los testimonios se presentan solo una vez al mes.

8. Solo el $50 \%$ dice que se lee a Ellen G. White en los temas centrales.

9. Más de un tercio afirma la existencia de programas paralelos al programa Culto Joven.

10. Más de la mitad de las iglesias afirma que el pastor predica en el Culto Joven solo una vez al mes.

\section{Conclusiones}

De acuerdo a los resultados observados, se puede concluir que 
las iglesias adventistas de la APCE del territorio de Lima desarrollan el programa del culto joven los sábados de tarde con alabanzas, testimonios y temas inspiradores, con los minutos apropiados para su realización, con una asistencia mayoritaria especialmente en las iglesias más pequeñas, incluso a pesar de que la mitad de ellas realizan el programa compitiendo con otras reuniones paralelas que interrumpen y afectan este importante culto. También se puede concluir que el pastor de las iglesias encuestadas no predica mucho en estos programas, lo que indica, que este -el pastor- le da al culto joven poca importancia.

De acuerdo al nuevo lanzamiento realizado por la DSA, el año 2013, todavía más de la mitad de las iglesias no han implementado el programa Culto Joven conforme lo recomienda el voto.

El nuevo planteamiento, para el programa Culto Joven, no responde a un Modelo de Culto Joven, debido a lo cual se hace imperativo trabajar en un modelo que exponga la pedagogía, metodología, habilidades, valores, currícu- lo, bases axiológicas y epistemológicas, fundamentadas en la Biblia y respaldadas por los escritos de Ellen G. de White.

Como se puede notar, el programa propuesto por la DSA todavía presenta desafíos en su aplicación, por lo tanto, se debe continuar investigando con el objetivo de crear un modelo que permita el desarrollo espiritual de los jóvenes adventistas, como su preparación para el cumplimiento de la misión. 


\section{GUÍA DE TRANSLITERACIÓN PARA CARACTERES HEBREOS Y GRIEGOS}

Consonantes y vocales equivalentes para los caracteres hebreos:

\begin{tabular}{|c|c|c|c|c|}
\hline 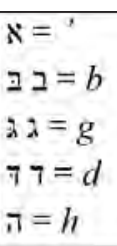 & $\begin{array}{l}T=w \\
T=z \\
ה=y \\
0=t \\
{ }^{\prime}=y\end{array}$ & $\begin{array}{l}2 \geq=k \\
y=1 \\
y=m i \\
\mathrm{~g}=n \\
0=s\end{array}$ & $\begin{array}{l}\mathrm{y}=t \\
\mathrm{y}=p \\
\mathrm{D}=f \\
\mathrm{y}=t s \\
\mathrm{P}=q\end{array}$ & $\begin{array}{l}7=r \\
\dot{U}=s \\
\mathbb{U}=s h \\
r=t \\
\pi=t h\end{array}$ \\
\hline $\begin{array}{l}=a \\
=a \\
=a\end{array}$ & $\begin{array}{l}y=e \\
y=\hat{y}=\hat{e} \\
0=e\end{array}$ & $\begin{array}{l}Q=i \\
Q=i\end{array}$ & $\begin{array}{l}y=0 \\
0=0 \\
j=0\end{array}$ & $\begin{array}{l}\vartheta=\hat{u} \\
q=u\end{array}$ \\
\hline
\end{tabular}

Consonantes y vocales equivalentes para los caracteres griegos:

\begin{tabular}{|l|l|l|l|l|}
\hline$\alpha=a$ & $\eta=\bar{e}$ & $v=n$ & $\tau=t$ & $=h$ \\
$\beta=b$ & $\theta=t h$ & $\xi=x$ & $v=y$ & $\hat{\rho}=r h$ \\
$\gamma=g$ & $\mathrm{t}=i$ & $0=o$ & $\varphi=p h$ & $\gamma \chi=n j$ \\
$\delta=d$ & $\kappa=k$ & $\pi=p$ & $\chi=c h$ & $\gamma \xi=n x$ \\
$\varepsilon=e$ & $\lambda=1$ & $\rho=r$ & $\psi=p s$ & $\gamma \kappa=n k$ \\
$\zeta=z$ & $\mu=m$ & $\sigma \zeta=s$ & $\omega=\bar{o}$ & $\gamma \gamma=n g$ \\
\hline
\end{tabular}

